\title{
INDICADORES BIOLÓGICOS DA QUALIDADE DO SOLO EM SISTEMA AGROSSILVOPASTORIL NO NOROESTE DO ESTADO DE MINAS GERAIS
}

\author{
Biological indicators of soil quality in agricultural-forestry-pasture system in northwest \\ region of Minas Gerais state, Brazil
}

\author{
Cláudia Milene Nascente Neves ${ }^{2}$, Marx Leandro Naves Silva ${ }^{3}$, Nilton Curi ${ }^{4}$, Renato Luiz Grisi Macedo ${ }^{5}$, \\ Fátima Maria de Souza Moreira ${ }^{3}$, Alexandre Fonseca D’Andréa ${ }^{6}$
}

\begin{abstract}
RESUMO
Estudos de qualidade do solo são importantes para o entendimento da interação dessa com o ecossistema como um todo. Nesse contexto, os microrganismos do solo desempenham papel fundamental para manutenção e produtividade de vários agroecossistemas. Objetivou-se, neste trabalho avaliar os atributos biológicos (carbono da biomassa microbiana, respiração basal e quociente microbiano), além do teor de carbono orgânico, possíveis indicadores das alterações na qualidade do solo impostas pelos diferentes sistemas de manejo, em relação ao cerrado nativo, em sistema agrossilvopastoril, no noroeste de Minas Gerais. Foram coletadas amostras, nas profundidades de 0-5, 5-20 e 20-40 cm, de um Latossolo Vermelho Distrófico típico (LVd), entre os municípios de Vazante e Paracatu. Os sistemas foram selecionados segundo o histórico de uso: (1) CN- cerrado nativo (testemunha); (2) EA- eucalipto + arroz (ano zero do sistema agrossilvopastoril, plantados após o desmatamento da vegetação nativa de cerrado); (3) ES- eucalipto + soja (ano um do sistema, semeadura da soja em substituição ao arroz); (4) EP- eucalipto + pastagem (ano dois do sistema, com pastagem de Brachiaria brizantha Stapf., para a engorda do gado de corte); (5) EPG- eucalipto + pastagem + gado de corte (ano três do sistema, com a introdução do gado de corte); (6) PP- pastagem plantada de Brachiaria brizantha Stapf.; e (7) EC- eucalipto no sistema convencional no espaçamento de 2,0 x 3,0 m. O carbono orgânico e o carbono da biomassa microbiana apresentaram valores mais elevados na camada superficial $(0-5 \mathrm{~cm})$, em relação às demais, em todos os sistemas. O carbono orgânico mostrou alteração substancial em relação aos sistemas estudados e às profundidades, revelando seu potencial como indicador da qualidade do solo em termos de reflexos das modificações impostas pelo manejo. O teor de carbono da biomassa microbiana foi reduzido em todos sistemas estudados em relação ao cerrado nativo, em função da ação antrópica. Com o progresso do sistema agrossilvopastoril houve uma recuperação do carbono da biomassa microbiana. Não foi observada diferença significativa entre os sistemas de manejo e o cerrado nativo quanto ao quociente metabólico e à respiração basal.
\end{abstract}

Termos para indexação: Carbono da biomassa microbiana, sistemas de manejo, cerrado.

\section{ABSTRACT}

Soil quality studies are important for understanding its interaction with the ecosystem as a whole. In this context, the soil microorganisms play a fundamental role for the maintenance and productivity of various agricultural ecosystems. This work had the objective to evaluate the biological attributes (microbial biomass carbon, basal respiration, and microbial quotient), as well as the organic matter content, possible indicators of alterations in soil quality imposed by different management systems, in relation to native cerrado, in agricultural - forestry - pasture system, in northwest of Minas Gerais state, Brazil. Samples were collected at 05, 5-20, and 20-40 cm depths, from a typical Dystrophic Red Latosol (Oxisol), between Vazante and Paracatu counties. The systems were selected according to the history of use, as follows: (1) $\mathrm{CN}$ - native cerrado (reference); (2) EA - eucalyptus + rice (year zero of agricultural - forestry - pasture system, planted after removal of cerrado native vegetation); (3) EP - eucalyptus + soybeans (year one of system, soybeans sowing substituting rice); (4) - EP - ES - eucalyptus + pasture (year two of system, using Brachiaria Brizantha Stapf. for cattle raising); (5) - EPG - eucalyptus + pasture + cattle (year three of system, with introduction of cattle); (6) PP - pasture cultivated with Brachiaria Brizantha Stapf.; and (7) EC - eucalyptus under conventional system in $2 \times 3 \mathrm{~m}$ spacing. The organic carbon and the microbial biomass carbon presented higher values in the superficial layer $(0-5 \mathrm{~cm})$, in relation to the other depths, in all systems. The organic carbon showed substantial alteration in relation to the studied systems and depths assessed, revealing its potential as soil quality indicator concerning the modifications imposed by management. The microbial biomass carbon content decreased in all systems in comparison with native cerrado due to anthropic action. Along the progress of the agricultural forestry - pasture system, there was a recovering of the microbial biomass carbon. There was no statistical difference between management systems and native cerrado concerning metabolic quotient and basal respiration.

Index terms: Microbial biomass carbon, management systems, cerrado.

(Recebido em 27 de abril de 2006 e aprovado em 14 de setembro de 2007)

\footnotetext{
${ }^{1}$ Parte da Dissertação de Mestrado apresentada pelo primeiro autor à Universidade Federal de Lavras - UFLA

'Zootecnista, Doutoranda em Solos e Nutrição de Plantas - Laboratório de Biogeoquímica Ambiental/CENA - Escola Superior de Agricultura "Luiz de Queiroz"/ESALQ - Universidade de São Paulo/USP - Avenida Pádua Dias 11 - Cx. P. 9 - 13418-900 - Piracicaba, SP - cmneves@esalq.usp.br ${ }^{3}$ Engenheiros Agrônomos, Doutores em Ciência do Solo, Professores - Departamento de Ciência do Solo/DCS - Universidade Federal de Lavras/UFLA Cx.P. 3037 - 37200-000 - Lavras, MG - marx@ufla.br - fmoreira@ufla.br - Bolsista do CNPq

${ }^{4}$ Engenheiro Agrônomo, PhD em Ciência do Solo, Professor - Departamento de Ciência do Solo/DCS - Universidade Federal de Lavras/UFLA - Cx. P. 3037 -

37200-000 - Lavras, MG - niltcuri@ufla.br - Bolsista do CNPq
${ }^{5}$ Engenheiro Florestal, Doutor em Engenharia Florestal, Professor - Departamento de Ciência Florestal/DCF - Universidade Federal de Lavras/UFLA - CX. ${ }^{5}$ Engenheiro Florestal, Doutor em Engenharia Florestal
P. 3037 - 37200-000 - Lavras, MG - rgrisi@ufla.br

${ }^{6}$ Engenheiro Agrônomo, Doutor em Solo e Nutrição de Plantas, Professor - Escola Agrotécnia Federal de Muzambinho/EAFM - Gabinete da Diretoria Geral - Rodovia Muzambinho, Km 35 - 37890-000 - Muzambinho, MG - alexdandrea@gmail.com
}

Ciênc. agrotec., Lavras, v. 33, n. 1, p. 105-112, jan./fev., 2009 


\section{INTRODUÇÃO}

São encontradas na literatura várias definições sobre qualidade do solo. Larson \& Pierce (1994) a definem como a capacidade do solo funcionar dentro dos limites do ecossistema e interagir positivamente com o ambiente externo. Para esses autores, a qualidade do solo varia consideravelmente, tanto em pequena quanto em grande escala, apresentando caráter dinâmico, podendo ser diminuída, mantida ou aumentada, e expressa tanto pelos atributos inerentes ao solo como pela habilidade desse em interagir com estímulos aplicados.

O conceito de qualidade do solo mais largamente aceito, segundo Nortcliff (2002), é com relação à produção agrícola. Nesse contexto, Karlen et al. (1997) definem qualidade de solo como sendo sua adaptação para funcionar com o ambiente, sustentar a produtividade vegetal e animal, manter ou melhorar a qualidade da água e do ar, e sustentar a sobrevivência do homem. Doran \& Parkin (1994) afirmam que a qualidade do solo descreve a capacidade desse para exercer funções de produção biológica, qualidade do ambiente e promover saúde das plantas e dos animais de maneira sustentável.

A manutenção da produtividade dos ecossistemas florestais e agrícolas depende, em grande parte, do processo de transformação da matéria orgânica e, conseqüentemente, da participação da biomassa microbiana do solo. Ela representa importante indicador ecológico, pois é responsável pela decomposição e mineralização dos resíduos vegetais e animais no solo. A biomassa microbiana é considerada como um reservatório de nutrientes e de energia potencialmente disponível para as plantas (JENKINSON \& LADD, 1981). Ela é fundamental na manutenção e produtividade de vários ecossistemas naturais e de muitos agroecossistemas, os quais dependem, em grande parte, de processos mediados pelos microrganismos.

A importância ecológica da biomassa microbiana tem como principais destaques, além do armazenamento de nutrientes, o de servir como indicador rápido de mudanças no solo, quando a matéria orgânica é a ele incorporada, e indicador da sensibilidade da microbiota a interferências no sistema (GRISI, 1995). Práticas de manejo inadequadas podem levar a um rápido declínio deste carbono, colaborando para o aumento das emissões de gás carbônico $\left(\mathrm{CO}_{2}\right)$ à atmosfera (LAL, 1999). O carbono orgânico total do solo é, particularmente, útil à compreensão da ciclagem da matéria orgânica e também um eficiente indicador da qualidade do solo, pois representa fonte e dreno de nutrientes do ecossistema por meio da mineralização e imobilização, respectivamente.
Objetivou-se, no presente trabalho, avaliar atributos biológicos como possíveis indicadores das alterações na qualidade do solo impostas pelos diferentes sistemas de manejo, em relação ao cerrado nativo, em um sistema agrossilvopastoril, no noroeste de Minas Gerais.

\section{MATERIALE MÉTODOS}

O trabalho foi desenvolvido na fazenda Bom Sucesso de propriedade da Companhia Mineira de Metais (CMM), entre os municípios de Vazante e Paracatu, no noroeste do estado de Minas Gerais, na latitude sul $17^{\circ} 33^{\prime}$ e longitude oeste $46^{\circ} 42^{\prime}$. O solo é classificado como Latossolo Vermelho Distrófico típico ( $\mathrm{LVd}$ ), textura muito argilosa e o clima da região é do tipo Cwa, caracterizado pela temperatura do mês mais frio inferior a $18^{\circ} \mathrm{C}$, e a precipitação do mês mais seco menor do que $60 \mathrm{~mm}$, sendo que as precipitações médias anuais variam de 1.300 a 1.400 mm (NEVES, 2002). Os sistemas foram selecionados segundo o histórico de uso: (1) CN - cerrado nativo (testemunha); (2) EA - eucalipto + arroz (ano zero do sistema agrossilvopastoril, plantados após o desmatamento da vegetação nativa de cerrado e das operações de preparo do solo, com aração e gradagem, correção e fertilização com NPK); (3) ES - eucalipto + soja (ano um do sistema, semeadura da soja em substituição ao arroz, na entrelinha do eucalipto, após o preparo do solo com aração e gradagem seguido de adubação com NPK; (4) EP - eucalipto + pastagem (ano dois do sistema, com pastagem de Brachiaria brizantha, para a engorda do gado de corte. A semeadura da forrageira ocorreu após o preparo do solo e a fertilização com uma mistura de fosfato natural e superfosfato simples; e (5) EPG - eucalipto + pastagem + gado de corte (ano três do sistema, com a introdução do gado de corte) (NEVES, 2002).

A título de comparação, selecionou-se uma área de pastagem plantada de Brachiaria brizantha de longo uso, sob pastejo contínuo de gado bovino em regime extensivo e sem manejo da fertilidade do solo nos últimos 10 anos (6 - PP), e uma área de plantio de eucalipto no sistema convencional, no espaçamento de 2,0 x 3,0 m, sem manejo da fertilidade do solo e sem tratos silviculturais nos últimos 10 anos (7 - EC).

As amostragens de solo foram feitas em três profundidades (0-5, 5-20 e 20-40 cm), na entrelinha dos plantios. Em cada profundidade, para todos os sistemas de manejo, foram retiradas seis amostras simples, as quais constituíram três amostras compostas, cada uma constituindo uma repetição, não caracterizando repetição espacial, no sentido estrito de áreas distintas e casualizadas. As amostras foram acondicionadas em sacos 
plásticos, sendo protegidas da luz e mantidas em caixas térmicas, sendo transportadas para o laboratório no prazo máximo de 48 horas após a coleta, onde foram secas ao ar e passadas em peneira de malha de 2,0 $\mathrm{mm}$ (terra fina).

A análise granulométrica foi efetuada pelo método da pipeta (DAY, 1965), após dispersão da amostra com $\mathrm{NaOH} 1 \mathrm{~mol} \mathrm{~L}^{-1}$ e agitação rápida $(6.000 \mathrm{rpm})$ por 15 minutos. $\mathrm{O}$ pH em água e os componentes do complexo sortivo foram determinados de acordo com Embrapa (1997) e encontram-se na Tabela 1.

Avaliou-se o carbono orgânico total por oxidação a quente com dicromato de potássio e titulação com sulfato ferroso amoniacal (WALKLEY \& BLACK, 1934). Uma parte das amostras foi conservada a $4^{\circ} \mathrm{C}$ para determinação do carbono da biomassa microbiana (C-BM) e da taxa de respiração microbiana (respiração basal), estimada pelo $\mathrm{CO}_{2}$ liberado.

A determinação do carbono da biomassa microbiana (C-BM) foi realizada pelo método da fumigação-extração (VANCE et al., 1987). O princípio básico desse método é a extração do carbono microbiano após a morte dos microrganismos e ruptura da membrana celular pelo ataque com clorofórmio e liberação dos constituintes celulares. O carbono foi extraído utilizando-se $20 \mathrm{~g}$ de solo de amostras fumigadas e não fumigadas (controle), com $100 \mathrm{ml}$ de $\mathrm{K}_{2} \mathrm{SO}_{4}$ $0,5 \mathrm{~mol} \mathrm{~L}^{-1}$, após agitação. Com base no substrato extraído, foi feita a digestão de $8 \mathrm{ml}$ do mesmo com adição de $2 \mathrm{ml}$ de $\mathrm{K}_{2} \mathrm{Cr}_{2} \mathrm{O}_{7} 0,0667 \mathrm{~mol} \mathrm{~L}^{-1} \mathrm{e}$ de uma mistura de duas partes (10 ml) de $\mathrm{H}_{2} \mathrm{SO}_{4}$ concentrado e uma parte $(5 \mathrm{ml})$ de $\mathrm{H}_{3} \mathrm{PO}_{4}$ concentrado. Toda essa mistura foi aquecida em chapa por 5 minutos contados após aparecimento das primeiras bolhas de fervura e posteriormente resfriada acrescentando-se $10 \mathrm{ml}$ de água destilada.

A titulação foi feita com sulfato ferroso amoniacal $0,0333 \mathrm{~mol} \mathrm{~L}^{-1}$ usando fenil-alanina (1\%) como indicador. Foi usado o fator Kec de 0,30 para conversão do carbono extraído a carbono da biomassa microbiana. A relação CBM/CO foi calculada de acordo com Sparling (1982).

A taxa de respiração basal foi estimada pelo $\mathrm{CO}_{2}$ liberado a partir de $20 \mathrm{~g}$ de solo durante incubação por 72 horas, com extração por solução de $\mathrm{NaOH} 0,05 \mathrm{~mol} \mathrm{~L}^{-1} \mathrm{e}$ titulação com $\mathrm{HCl}$ 0,05 mol L-1 (ISERMEYER, 1952, citado por ALEF \& NANNIPIERI, 1995). O quociente metabólico $\left(q \mathrm{CO}_{2}\right)$ foi calculado pela divisão entre os valores da taxa de respiração basal pela biomassa microbiana (ANDERSON \& DOMSCH, 1993).

O delineamento experimental utilizado foi inteiramente casualizado (DIC), com estrutura em faixas de acordo com as profundidades de amostragem, onde as parcelas são os sistemas de manejo e a subparcelas são as profundidades. Os resultados foram submetidos à análise de variância, sendo o efeito da profundidade, dos sistemas de manejo e da interação sistema $\mathrm{x}$ profundidade comparados pelo teste de Scott-Knott (SCOTT \& KNOTT, 1974), a 5\% de probabilidade, para comparação entre as médias, utilizando-se o programa SISVAR (FERREIRA, 2000). O modelo seguido foi: $\mathrm{Yij}=\mathrm{m}+\mathrm{Si}+$ Erro (sistema) + Pj + SPij + Erro (geral), em que: Yij: valor de cada observação; m: média geral; Si: efeito do sistema i; Erro (sistema): erro devido ao efeito da repetição dentro do sistema i; Pj: efeito da profundidade j; SPij: efeito da interação entre o sistema i e a profundidade j; Erro (geral).

\section{RESULTADOS E DISCUSSÃO}

Os resultados das análises de $\mathrm{pH}$ em água e dos componentes do complexo sortivo podem ser observados na Tabela 1 .

Os resultados do carbono orgânico total $(\mathrm{CO})$ para os diferentes sistemas nas três profundidades encontramse na Tabela 2. Houve interação significativa entre sistemas de manejo e profundidades sobre os teores de carbono orgânico total do solo, com maiores teores de $\mathrm{CO}$ próximo à superfície, em todos os sistemas (Tabela 2 ). $\mathrm{O}$ valor de $\mathrm{CO}$ na camada superficial do cerrado nativo $(0-5 \mathrm{~cm})$ foi, em média, de 22,6 $\mathrm{g} \mathrm{kg}^{-1}$, e os menores valores obtidos foram nos sistemas EA, ES, EP e EPG, 13,5; 15,2; 14,8 e 14,6 $\mathrm{g} \mathrm{kg}^{-1}$, respectivamente. Valores intermediários foram encontrados apenas no eucalipto em sistema convencional, na ordem de $19,7 \mathrm{~g} \mathrm{~kg}^{-1}$.

Em todos os tratamentos estudados, houve uma tendência de diminuição nos teores de $\mathrm{CO}$ com o aumento da profundidade do solo (Tabela 2), com exceção do sistema PP nas duas primeiras profundidades de amostragem. Resultados semelhantes foram observados por Freixo et al. (2002), em estudo desenvolvido na região dos cerrados. Na camada superficial, os teores de CO foram mais elevados porque é onde os resíduos orgânicos são depositados, com maior formação de serrapilheira, e onde acontece maior atividade de microrganismos e também do sistema radicular das plantas. No cerrado nativo $(\mathrm{CN})$ esse acúmulo é mais evidente, possivelmente pela ausência de revolvimento, favorecendo assim, um maior acúmulo de matéria orgânica.

Depois do CN, os sistemas PP e EC apresentaram diferenças significativas e apresentaram valores intermediários de $\mathrm{CO}$ (Tabela 2), possivelmente por serem sistemas mais estabilizados, que estão instalados há vários anos. Onde não houve manejo que perturbasse esse equilíbrio, conseqüentemente ocorreu um maior acúmulo de matéria orgânica. 
Tabela 1 - Caracterização do Latossolo Vermelho Distrófico típico textura muito argilosa, para diferentes sistemas de manejo e profundidades.

\begin{tabular}{|c|c|c|c|c|c|c|c|c|c|c|}
\hline Sistemas & Prof. & $\mathrm{pH}$ & $\mathrm{P}$ & $\mathrm{K}$ & $\mathrm{Ca}$ & $\mathrm{Mg}$ & $\mathrm{Al}$ & SB & $\mathrm{V}$ & $\mathrm{m}$ \\
\hline & -- cm -- & & \multicolumn{2}{|c|}{--- $\mathrm{mg} \mathrm{dm}^{-3}$---- } & \multicolumn{4}{|c|}{ - $\mathrm{cmol}_{\mathrm{c}} \mathrm{dm}^{-3}$} & \multicolumn{2}{|c|}{-------\% ------ } \\
\hline \multirow{3}{*}{$\mathrm{CN}$} & $0-5$ & 4,8 & 1,00 & 76,67 & 0,57 & 0,17 & 1,50 & 0,93 & 11,30 & 62,00 \\
\hline & $5-20$ & 4,9 & 0,53 & 53,00 & 0,40 & 0,17 & 1,33 & 0,70 & 9,77 & 65,33 \\
\hline & $20-40$ & 4,7 & 1,40 & 41,67 & 0,43 & 0,20 & 1,27 & 0,73 & 12,40 & 62,33 \\
\hline \multirow{3}{*}{ EA } & $0-5$ & 5,2 & 0,37 & 40,67 & 1,40 & 1,33 & 0,20 & 2,83 & 44,77 & 8,00 \\
\hline & $5-20$ & 4,9 & 0,30 & 24,67 & 0,77 & 0,23 & 0,50 & 1,10 & 20,53 & 33,33 \\
\hline & $20-40$ & 4,9 & 0,10 & 16,33 & 0,43 & 0,17 & 0,47 & 0,63 & 15,20 & 42,33 \\
\hline \multirow{3}{*}{ ES } & $0-5$ & 5,7 & 0,73 & 52,33 & 2,57 & 2,43 & 0,00 & 5,13 & 67,77 & 0,00 \\
\hline & $5-20$ & 5,0 & 0,47 & 30,33 & 0,67 & 0,20 & 0,77 & 0,97 & 18,53 & 44,67 \\
\hline & $20-40$ & 4,9 & 0,27 & 23,00 & 0,43 & 0,17 & 0,53 & 0,63 & 15,90 & 45,33 \\
\hline \multirow{3}{*}{ EP } & $0-5$ & 5,2 & 4,83 & 40,67 & 1,47 & 1,30 & 0,33 & 2,87 & 41,37 & 11,33 \\
\hline & $5-20$ & 5,2 & 1,93 & 23,67 & 0,87 & 0,47 & 0,87 & 1,43 & 22,90 & 40,33 \\
\hline & $20-40$ & 5,1 & 0,30 & 18,00 & 0,43 & 0,20 & 1,07 & 0,67 & 12,73 & 61,00 \\
\hline \multirow{3}{*}{ EPG } & $0-5$ & 5,5 & 0,60 & 42,33 & 1,47 & 1,40 & 0,17 & 3,00 & 46,13 & 5,33 \\
\hline & $5-20$ & 5,2 & 0,20 & 17,00 & 0,77 & 0,20 & 0,73 & 1,00 & 20,10 & 41,00 \\
\hline & $20-40$ & 5,1 & 0,20 & 14,67 & 0,53 & 0,17 & 0,73 & 0,70 & 15,97 & 50,67 \\
\hline \multirow{3}{*}{ PP } & $0-5$ & 5,5 & 0,80 & 157,67 & 1,03 & 0,80 & 0,30 & 2,23 & 36,60 & 12,00 \\
\hline & $5-20$ & 5,8 & 0,37 & 41,33 & 0,73 & 0,27 & 0,67 & 1,13 & 20,53 & 38,00 \\
\hline & $20-40$ & 5,7 & 0,20 & 22,33 & 0,50 & 0,17 & 0,67 & 0,77 & 14,80 & 47,00 \\
\hline \multirow{3}{*}{$\mathrm{EC}$} & $0-5$ & 4,9 & 2,10 & 62,33 & 0,77 & 0,20 & 1,40 & 1,13 & 14,30 & 55,33 \\
\hline & $5-20$ & 5,0 & 1,10 & 29,33 & 0,50 & 0,20 & 1,43 & 0,80 & 11,77 & 65,00 \\
\hline & $20-40$ & 5,0 & 0,60 & 23,67 & 0,40 & 0,20 & 1,33 & 0,70 & 12,40 & 67,00 \\
\hline
\end{tabular}

CN: cerrado nativo; EA: eucalipto+arroz; ES: eucalipto+soja; EP: eucalipto+pastagem; EPG: eucalipto+pastagem+gado de corte; PP: pastagem plantada: EC: eucalipto convencional. SB: soma de bases; V: saturação por bases; m: saturação por Al.

Tabela 2 - Carbono orgânico total do Latossolo Vermelho Distrófico típico, para diferentes profundidades e sistemas de manejo e profundidades.

\begin{tabular}{cccc}
\hline \multirow{2}{*}{ Sistemas } & \multicolumn{3}{c}{ Profundidades $(\mathrm{cm})$} \\
\cline { 2 - 4 } & $0-5$ & $5-20$ & $20-40$ \\
\hline & $-----------\mathrm{g} \mathrm{kg}^{-1}$ & $11,9 \mathrm{a} \mathrm{C}$ \\
$\mathrm{CN}$ & $22,6 \mathrm{a} \mathrm{A}$ & $15,2 \mathrm{a} \mathrm{B}$ & $8,5 \mathrm{~b} \mathrm{C}$ \\
EA & $13,5 \mathrm{c} \mathrm{A}$ & $11,4 \mathrm{c} \mathrm{B}$ & $8,3 \mathrm{~b} \mathrm{C}$ \\
ES & $15,2 \mathrm{c} \mathrm{A}$ & $11,6 \mathrm{c} \mathrm{B}$ & $9,1 \mathrm{~b} \mathrm{C}$ \\
EP & $14,8 \mathrm{c} \mathrm{A}$ & $12,5 \mathrm{c} \mathrm{B}$ & $8,9 \mathrm{~b} \mathrm{~B}$ \\
EPG & $14,6 \mathrm{c} \mathrm{A}$ & $10,6 \mathrm{c} \mathrm{B}$ & $11,2 \mathrm{a} \mathrm{B}$ \\
PP & $15,4 \mathrm{c} \mathrm{A}$ & $14,5 \mathrm{a} \mathrm{A}$ & $11,2 \mathrm{a} \mathrm{C}$ \\
EC & $19,7 \mathrm{~b} \mathrm{~A}$ & $13,3 \mathrm{~b} \mathrm{~B}$ &
\end{tabular}

$\mathrm{CN}$ : cerrado nativo; EA: eucalipto+arroz; ES: eucalipto+soja; EP: eucalipto+pastagem; EPG: eucalipto+pastagem+gado de corte; PP: pastagem plantada: EC: eucalipto convencional. Médias seguidas pela mesma letra, minúscula na coluna e maiúscula na linha, não diferem estatisticamente entre si pelo teste de ScottKnott, a $5 \%$ de probabilidade.
Exceto na profundidade de $0-5 \mathrm{~cm}$, do sistema PP (Tabela 2), esse efeito pode ser devido ao grau de degradação que a pastagem se encontrava, onde segundo observações de campo, encontravam-se sítios de encrostamento superficial, erosão laminar, grande incidência de plantas invasoras e pragas (NEVES, 2002). Já nas profundidades de $5-20$ e $20-40 \mathrm{~cm}$, o teor de CO do sistema PP se iguala estatisticamente com o teor do sistema $\mathrm{CN}$ (Tabela 2), o que pode ser devido à alta densidade do sistema radicular da gramínea, o que está de acordo com Greenland (1971). Segundo esse autor, a recuperação dos teores de $\mathrm{CO}$ pode ser obtida através da implantação de pastagem, ou, no caso de sistemas de produção intensivos, da redução do revolvimento do solo e da adoção de um sistema de rotação de culturas com a inclusão de espécies de alta produção de fitomassa (BAYER \& MIELNICZUK, 1997).

Foi também observado um declínio relativo nos teores de $\mathrm{CO}$ quando se passa do $\mathrm{CN}$ para o sistema agrossilvopastoril (Tabela 2). Possivelmente, isso ocorreu pelo fato da retirada de nutrientes pela exportação de grãos 
nos primeiros anos (EA - arroz e ES - soja), que deve ter reduzido os estoques orgânicos do solo antes que houvesse um ganho líquido de matéria orgânica em decorrência do manejo integrado. Não foi possível observar uma tendência de aumento no teor de CO, com o passar dos anos, no sistema agrossilvipastoril (sentido EP e EPG), talvez pelo pequeno tempo decorrido desde a sua implantação e a adubação com N, P e K. Desse modo, a adoção de sistemas de uso e manejo do solo deve levar em consideração, entre outros aspectos, o seu efeito sobre os teores de CO.

Os resultados do carbono da biomassa microbiana (C-BM), para os diferentes sistemas de manejo nas três profundidades, são apresentados na Tabela 3. Houve interação significativa entre sistemas de manejo e profundidades sobre os teores de carbono da biomassa microbiana do solo.

$\mathrm{O}$ valor de C-BM na camada superficial do cerrado nativo $(0-5 \mathrm{~cm})$ foi, em média, de $1495 \mu \mathrm{g} \mathrm{g}^{-1}$, valores de $\mathrm{C}$ $\mathrm{BM}$ na ordem de $1310 \mu \mathrm{g} \mathrm{g}^{-1}$ foram observados por D’Andréa et al. (2002), e os menores valores de carbono da biomassa microbiana foram obtidos dentro do sistema agrossilvopastoril (EA, ES, EP, EPG), 494,0; 775,4; 800,1 e 763,5 $\mu_{\mathrm{g} \mathrm{g}^{-1}}$, respectivamente. Os valores de C-BM nos sistemas convencionais não diferiram daqueles do cerrado nativo, e foram, respectivamente, de 1211,8 e 1272,4 $\mu \mathrm{g} \mathrm{g} \mathrm{g}^{-1}$, para PP e EC, nesta ordem. Os valores de carbono da biomassa microbiana foram significativos em relação às profundidades estudadas, exceto para os sistemas EA e EPG.

Tabela 3 - Carbono da biomassa microbiana do Latossolo Vermelho Distrófico típico, para diferentes sistemas de manejo e profundidades.

\begin{tabular}{cccc}
\hline \multirow{2}{*}{ Sistemas } & \multicolumn{3}{c}{ Profundidades $(\mathrm{cm})$} \\
\cline { 2 - 4 } & $0-5$ & $5-20$ & $20-40$ \\
\hline & $--------------\mu^{-1}$ g & \\
CN & 1495,6 a A & 1083,0 a B & 683,4 a C \\
EA & 494,0 b A & 677,7 b A & 437,8 a A \\
ES & 775,4 b B & 1142,1 a A & 805,6 a B \\
EP & 800,1 b B & 1175,7 a A & 723,0 a B \\
EPG & 763,5 b A & 963,9 a A & 735,5 a A \\
PP & 1211,8 a A & 768,7 b B & 923,8 a B \\
EC & 1272,4 a A & 1037,1 a A & 662,0 a B \\
\hline
\end{tabular}

CN: cerrado nativo; EA: eucalipto+arroz; ES: eucalipto+soja; EP: eucalipto+pastagem; EPG: eucalipto+pastagem+gado de corte; PP: pastagem plantada: EC: eucalipto convencional. Médias seguidas pela mesma letra, minúscula na coluna e maiúscula na linha, não diferem estatisticamente entre si pelo teste de Scott-Knott, a 5\% de probabilidade.
De modo geral, houve tendência dos maiores valores ocorrerem nas camadas superiores em relação à camada inferior, devido às condições mais desfavoráveis ao desenvolvimento dos microrganismos e pela menor disponibilidade de matéria orgânica nessa última. As maiores diferenças foram encontradas nos sistemas $\mathrm{CN}$, PP e EC (embora não significativas), que são sistemas mais estáveis. Já nos sistemas EA, ES, EP e EPG os maiores valores de $\mathrm{C}$-BM foram registrados na profundidade de 5$20 \mathrm{~cm}$. Isso pode ser devido, possivelmente, à incorporação de resíduos orgânicos ao solo por ocasião do preparo e colheita.

Em estudo envolvendo sistemas convencionais e de plantio direto na região dos cerrados no Sul de Goiás, d'Andréa (2001) encontrou redução do C-BM em profundidade somente no cerrado nativo. $\mathrm{O}$ mesmo resultado foi obtido por Silva (2001), em estudo na região dos Campos das Vertentes, em Minas Gerais.

Observa-se uma queda significativa do C-BM quando se passa do $\mathrm{CN}$ para os sistemas de cultivo, com exceção do EC. O mesmo comportamento foi observado por Matsuoka et al. (2003), em estudo de sistemas de uso do solo com culturas perenes e anuais, em relação à área sob vegetação nativa. Não houve um comportamento sistemático de aumento do teor de C-BM com o passar dos anos nos sistemas EA, ES, EP e EPG. Segundo Mendes et al. (1999), à medida que os solos sob cerrado são incorporados aos processos agrícolas, principalmente aos convencionais, ocorre uma queda acentuada nos níveis da biomassa ao longo dos anos. Isso ocorre em função da crescente demanda energética da população microbiana em adaptação às novas condições e à redução nas adições de matéria orgânica. Depois de cessado o efeito inicial promovido pelo desmatamento e a capacidade de deposição for aumentada pelo crescimento da cultura ou por práticas culturais, novo equilíbrio, com teores mais elevados de biomassa microbiana, tende a ocorrer.

A relação C-BM/CO seguiu a mesma tendência apresentada pelos resultados de carbono da biomassa microbiana, principalmente na camada superficial do solo (Tabela 4). A contribuição do carbono da biomassa microbiana para o carbono orgânico total do solo variou, na camada de $0-5 \mathrm{~cm}$, de 3,6 a 7,9\%. O maior valor, referente à pastagem plantada, destacou-se dos demais sistemas apesar de não diferir estatisticamente dos sistemas cerrado (CN) e floresta plantada de eucalipto (EC) (Tabela 4). Resultados semelhantes foram observados em estudo desenvolvido na Nova Zelândia, o qual evidenciou uma relação C-BM/CO maior em pastagens, quando comparadas com floresta nativa e floresta plantada com 
espécies exóticas (SPARLING, 1992). No Brasil, experimentos realizados na região Amazônica revelaram relação C-BM/CO maior na pastagem de quatro anos do que na floresta nativa (GERALDES et al., 1995). No entanto, com o aumento do tempo de estabelecimento da pastagem, os autores observaram um decréscimo nessa relação, indicando redução da participação do carbono da biomassa microbiana em relação ao carbono orgânico total.

Tabela 4 - Relação carbono da biomassa microbiana e carbono orgânico total do Latossolo Vermelho Distrófico típico, para diferentes sistemas de manejo e profundidades.

\begin{tabular}{cccc}
\hline \multirow{2}{*}{ Sistemas } & \multicolumn{3}{c}{ Profundidades (cm) } \\
\cline { 2 - 4 } & $0-5$ & $5-20$ & $20-40$ \\
\cline { 2 - 4 } & ------------ \% -------- \\
\hline CN & 6,6 a A & 7,1 a A & 5,7 b B \\
EA & 3,6 b B & 5,9 b A & 5,1 b A \\
ES & 5,1 b B & 9,8 a A & 9,7 a A \\
EP & 5,4 b B & 9,4 a A & 7,9 a A \\
EPG & 5,2 b B & 9,1 a A & 6,5 b B \\
PP & 7,9 a A & 5,3 b B & 8,2 a A \\
EC & 6,4 a B & 7,8 a A & 5,9 b B \\
\hline
\end{tabular}

CN: cerrado nativo; EA: eucalipto+arroz; ES: eucalipto+soja; EP: eucalipto+pastagem; EPG: eucalipto+pastagem+gado de corte; PP: pastagem plantada: EC: eucalipto convencional. Médias seguidas pela mesma letra, minúscula na coluna e maiúscula na linha, não diferem estatisticamente entre si pelo teste de ScottKnott, a 5\% de probabilidade.

Com relação ao sistema agrossilvopastoril (EA, ES, $\mathrm{EP}, \mathrm{EPG})$, foram observadas relações C-BM/CO menores em comparação aos outros sistemas (CN, PP e EC) e uma tendência de aumento da relação C-BM/CO após o estabelecimento dos sistemas de pastagens (EP e EPG). A recuperação dos teores de carbono da biomassa microbiana após o reestabelecimento da pastagem nas áreas de cultivo anual (EA e ES) foi rápida em razão dos baixos teores de carbono orgânico total (Tabela 2), a relação C-BM/CO atingiu os níveis mais elevados após um ano, concordando com os resultados observados por Sparling (1992). A queda no valor da relação C-BM/CO observada no sistema APG, possivelmente está relacionada ao pisoteio dos animais e suas conseqüências (GERALDES et al., 1995). Houve uma tendência de elevação dos valores da relação C-BM/CO em profundidade, possivelmente devido, aos baixos teores de carbono orgânico e maiores teores de carbono da biomassa microbiana.

A relação C-BM/CO é influenciada por diversos fatores. Entre eles podem ser citados o grau de estabilização do carbono orgânico e o histórico de manejo do solo. Para se saber o quanto a relação C-BM/CO está em equilíbrio ou se encontra ainda em fase de degradação ou recuperação, é necessário estabelecer um valor de referência para cada situação particular, sendo, portanto, pouco extrapolável para outras situações (D’ANDRÉA et al., 2002; SPARLING 1995).

A respiração basal representa a oxidação da matéria orgânica por organismos aeróbios do solo e o $q \mathrm{CO}_{2}$ expressa a quantidade de carbono oxidado por grama de carbono da biomassa microbiana por certo tempo. Os resultados de respiração basal e do $q \mathrm{CO}_{2}$ para os diferentes sistemas de manejo e profundidades estão apresentados na Tabela 5 . Não foram verificadas diferenças significativas nos valores de respiração basal e o $q \mathrm{CO}_{2}$ entre os sistemas de manejo e as profundidades. O mesmo foi observado por D'Andréa et al. (2002), que também não encontraram diferenças nesses atributos em sistema de plantio direto e plantio convencional quando comparados com o cerrado nativo.

Tabela 5 - Respiração basal e quociente metabólico do Latossolo Vermelho Distrófico típico, para diferentes profundidades e sistemas de manejo.

\begin{tabular}{|c|c|c|c|}
\hline \multirow{3}{*}{ Sistemas } & \multicolumn{3}{|c|}{ Profundidades $(\mathrm{cm})$} \\
\hline & $0-5$ & $5-20$ & $20-40$ \\
\hline & \multicolumn{3}{|c|}{ Respiração basal, $\mu \mathrm{g} \mathrm{C}-\mathrm{CO}_{2} \mathrm{~g}^{-1} \mathrm{~h}^{-1}$} \\
\hline $\mathrm{CN}$ & 18,62 & 17,47 & 15,67 \\
\hline EA & 18,47 & 24,59 & 16,59 \\
\hline ES & 17,51 & 16,27 & 12,07 \\
\hline EP & 15,33 & 15,06 & 14,05 \\
\hline EPG & 21,08 & 21,28 & 12,50 \\
\hline $\mathrm{PC}$ & 19,30 & 21,21 & 12,26 \\
\hline $\mathrm{EC}$ & 17,90 & 19,28 & 18,55 \\
\hline \multirow[t]{2}{*}{ Média } & 16,12 & 19,31 & 14,53 \\
\hline & \multicolumn{3}{|c|}{$q \mathrm{CO}_{2}, \mu \mathrm{g} \mathrm{C}-\mathrm{CO}_{2} \mu \mathrm{g} \mathrm{C}-\mathrm{CBM}^{-1} \mathrm{dia}^{-1}$} \\
\hline $\mathrm{CN}$ & 0,30 & 0,39 & 0,55 \\
\hline EA & 0,90 & 0,87 & 0,91 \\
\hline ES & 0,54 & 0,34 & 0,36 \\
\hline EP & 0,46 & 0,31 & 0,47 \\
\hline EPG & 0,66 & 0,53 & 0,41 \\
\hline $\mathrm{PC}$ & 0,38 & 0,66 & 0,32 \\
\hline $\mathrm{EC}$ & 0,34 & 0,45 & 0,67 \\
\hline Média & 0,51 & 0,51 & 0,53 \\
\hline
\end{tabular}

CN: cerrado nativo; EA: eucalipto+arroz; ES: eucalipto+soja; EP: eucalipto+pastagem; EPS: eucalipto+pastagem+gado de corte; PP: pastagem plantada: EC: eucalipto convencional. Médias seguidas pela mesma letra, minúscula na linha, não diferem estatisticamente entre si pelo teste de Scott-Knott, a $5 \%$ de probabilidade. 
Em sistemas de manejo com menor revolvimento do solo, existem melhores condições para o desenvolvimento das populações microbianas na camada superficial do solo do que nos sistemas com maior revolvimento (DORAN, 1980). Os sistemas com revolvimento sistemático do solo provocam perturbações promotoras de estresse na população microbiana e, uma vez que as adições de carbono nesses sistemas são menores, os microrganismos terminam por consumir o carbono orgânico do solo, causando sua redução (D’ANDRÉA et al., 2002). Assim, maiores valores de $q \mathrm{CO}_{2}$ indicam que a população microbiana está consumindo mais carbono oxidável para sua manutenção (ANDERSON \& DOMSCH, 1993;DIAS-JÚNIOR et al., 1998).

Embora não tenha havido diferenças significativas entre os sistemas para a respiração basal e o $q \mathrm{CO}_{2}, o q \mathrm{CO}_{2}$ apresentou o maior valor nos sistemas EA, ES, EP e EPG, em média, cerca de duas vezes maior do que verificado nos sistemas sem revolvimento (CN, PC e EC), indicando uma situação de estresse naqueles sistemas. Nas três primeiros situações (EA, ES e EP), devido ao revolvimento do solo para o plantio de arroz, da soja e da braquiária e no caso do EPG, devido ao pisoteio do gado (Tabela 5).

Vale destacar que os sistemas avaliados ainda são recentes e que a biomassa microbiana pode estar ainda em adaptação às condições do solo sob manejo. Por fim, devese levar em consideração que apenas 15 a $30 \%$ da biomassa microbiana do solo é ativa (MACDONALD, 1986) e que o restante dos microrganismos do solo ocorre em formas latentes ou inativas, com baixa atividade metabólica (MOREIRA \& SIQUEIRA, 2002), o que pode causar dificuldades adicionais na interpretação dos resultados do $q \mathrm{CO}_{2}$, já que em seu cálculo é levado em conta o teor total de carbono da biomassa microbiana do solo (D'ANDRÉA et al., 2002).

\section{CONCLUSÕES}

O estoque de carbono orgânico mostrou alteração em relação aos sistemas estudados e às profundidades, revelando seu potencial como indicador da qualidade do solo refletindo as modificações impostas pelo manejo.

O teor de carbono da biomassa microbiana foi reduzido em todos sistemas estudados em relação ao cerrado nativo, em função da ação antrópica. Com o progresso do sistema agrossilvopastoril houve uma recuperação do carbono da biomassa microbiana.

Não foi observada diferença significativa entre os sistemas de manejo e o cerrado nativo quanto ao quociente metabólico e à respiração basal.

\section{REFERÊNCIAS BIBLIOGRÁFICAS}

ALEF, K.; NANNIPIERI, P. (Eds.). Methods in applied soils microbiology and biochemistry. London: Academic, 1995. $576 \mathrm{p}$.

ANDERSON, T. H.; DOMSCH, K. H. The metabolic quocient for $\mathrm{CO}_{2}\left(\mathrm{qCO}_{2}\right)$ as a specific activity parameter to assess the effects of environmental conditions, such as $\mathrm{pH}$, on the microbial biomass of forest soil. Soil Biology and Biochemistry, Oxford, v. 25, n. 3, p. 393-395, Mar. 1993.

BAYER, C.; MIELNICZUK, J. Características químicas do solo afetadas por métodos de preparo e sistemas de culturas. Revista Brasileira de Ciência do Solo, Campinas, v. 21, p. 105-112, 1997.

D'ANDRÉA, A. F.; SILVA, M. L. N.; CURI, N.; SIQUEIRA, J. O.; CARNEIRO, M. A. C. Atributos biológicos indicadores da qualidade do solo em sistemas de manejo na região do cerrado no sul do Estado de Goiás. Revista Brasileira de Ciência do Solo, Viçosa, v. 26, n. 4, p. 913923, 2002.

DAY, P. R. Particle fractionation and particle-size analysis. In: BLAKE, C. A. Methods of soil analysis. Madison: American Society of Agronomy, 1965. pt. 1, p. 545-566.

DIAS-JÚNIOR, H. E.; MOREIRA, F. M. S.; SIQUEIRA, J. O.; SILVA, R. Metais pesados, densidade e atividade microbiana em solo contaminado por rejeitos de indústria de zinco. Revista Brasileira de Ciência do Solo, Viçosa, v. 22, n. 4, p. 631-640, 1998.

DORAN, J. W.; PARKIN, T. B. Defining and assessing soil quality. In: DORAN, J. W.; COLEMAN, D. C.; BEZDICEK, D. F.; STEWART, B. A. (Eds.). Defining soil quality for a sustainable environment. Madison: Soil Science Society of America, 1994. p. 3-21. (SSSA special publication, 35).

EMPRESA BRASILEIRA DE PESQUISA AGROPECUÁRIA. Manual de métodos de análises de solo. Rio de Janeiro: Ministério da Agricultura, 1997. Não paginado.

FERREIRA, D. F. Análises estatísticas por meio do SISVAR (Sistema para análise de variância) para Windows versão 4.0. In: REUNIÃO ANUAL DA REGIÃO BRASILEIRA DA SOCIEDADE INTERNACIONAL DE BIOMETRIA, 45., 2000, São Carlos. Anais... São Carlos: UFSCar, 2000. p. 255-258. 
FREIXO, A. A.; MACHADO, P. L. O. A.; GUIMARÃES, C. M.; SILVA, C. A.; FADIGAS, F. S. Estoque de carbono e nitrogênio e distribuição de frações orgânicas de Latossolo do cerrado sob diferentes sistemas de cultivo. Revista Brasileira de Ciência do Solo, Viçosa, v. 26, n. 2, p. 425434, 2002.

GERALDES, A. P. A.; CERRI, C. C.; FEIGL, B. J. Biomassa microbiana de solo sob pastagens na Amazônia. Revista Brasileira de Ciência do Solo, Campinas, v. 19, p. 55-60, 1995.

GREENLAND, D. J. Change in the nitrogen status and physical condition of soils under pastures, with special reference to the maintenance of the fertility of Australian soils used for growing wheat. Soil and Fertilizers, Berlin, v. 34, p. 237-251, 1971.

GRISI, B. M. Biomassa e atividade de microrganismos do solo: revisão metodológica. Revista Nordestina de Biologia, João Pessoa, v. 10, n. 1, p. 1-22, 1995.

JENKINSON, D. D.; LADD, L. N. Microbial biomass in soil: measurement and turnover. In: PAUL, E. A.; LADD, J. N. (Eds.). Soil biochemistry. New York: M. Dekker, 1981. v. 5, p. 415-471.

KARLEN, D. L.; MAUSBACH, M. J.; DORAN, J. W.; CLINE, R. G.; HARRIS, R. F.; SCHUMAN, G. E. Soil quality: a concept, definition and framework for valuation. Soil Science Society of America, Madison, v. 61, n. 1, p. 4-10, Jan./Feb. 1997.

LAL, R. World soils and the greehouse effect. Global Change Newsletter, Stockoholm, n. 37, p. 4-5, Mar. 1999.

LARSON, W. E.; PIERCE, F. J. The dynamics of soil quality as a measure of sustainable management. In: DORAN, J. W.; COLEMAN, D. C.; BEZDICEK, D. F.; STEWART, B. A. (Eds.). Defining soil quality for a sustainable environment. Madison: Soil Science Society of America, 1994. p. 37-51. (SSSA special publication, 35).

MacDONALD, R. M. Extration of microorganisms from soil. Biological Agriculture Horticulture, Bicester, v. 3, n. 4, p. 361-365, 1986.

MATSUOKA, M.; MENDES, I. C.; LOUREIRO, M. F. Biomassa microbiana e atividade enzimática em solos sob vegetação nativa e sistemas agrícolas anuais e perenes na região de Primavera do Leste (MT). Revista Brasileira de Ciência do Solo, Viçosa, v. 27, n. 3, p. 425-433, maio/jun. 2003.

MENDES, I. C.; VIVALDI, L.; RIBEIRO, J. A.; VARGAS, M. A. T.; RIBEIRO, F. J. Biomassa -C e atividade microbiana em solos do bioma cerrado sob vegetação nativa. Brasília, DF: Embrapa-CPAC, 1999. v. 4.

MOREIRA, F. M. S.; SIQUEIRA, J. O. Microbiologia e bioquímica do solo. Lavras: UFLA, 2002. 625 p.

NEVES, C. M. N. Atributos indicadores da qualidade do solo em sistema agrossilvopastoril, no noroeste do estado de Minas Gerais. 2002. 87 p. Dissertação (Mestrado em Solos e Nutrição de Plantas) - Universidade Federal de Lavras, Lavras, 2002.

NORTCLIFF, S. Standardisation of soil quality attributes. Agriculture, Ecosystems \& Environment, Amsterdam, v. 88, n. 2. p. 161-168, Feb. 2002.

SCOTT, A. J.; KNOTT, M. Accouter analysis methods for grouping means in the analysis of variants. Biometrics, Saint Louis, n. 30, n. 3, p. 507-512, Sept. 1974.

SILVA, R. R. Qualidade do solo em função de diferentes sistemas de manejo na região Campos das Vertentes, Bacia Alto Rio Grande - MG. 2001. 97 p. Dissertação (Mestrado em Solos e Nutrição de Plantas) - Universidade Federal de Lavras, Lavras, 2001.

SPARLING, G. P. Ratio of microbial biomass carbon to soil organic carbon as a sensitive indicator of changes in soil organic matter. Australian Journal of Soil Research, Collingwood, v. 30, n. 2, p. 195-207, 1992.

VANCE, E. D.; BROOKES, P. C.; JENKINSON, D. S. An extraction method for measuring soil microbial biomass $\mathrm{C}$. Soil Biology Biochemistry, Oxford, v. 19, n. 6, p. 703-707, 1987.

WALKLEY, A.; BLACK, I. A. An examination of the Degtjareff method for determining soil organic matter and a proposed modification of the chromic acid titration method. Soil Science, Baltimore, v. 37, n. 1, p. 29-38, Jan./ June 1934. 